\title{
Two-dimensional Array Beam Scanning via Externally and Mutually Injection Locked Coupled Oscillators
}

\author{
Ronald J. Pogorzelski \\ Jet Propulsion Laboratory \\ California Institute of Technology \\ Pasadena, CA 91109
}

\section{Background}

Some years ago, Stephan [1] proposed an approach to one dimensional (linear) phased array beam steering which requires only a single phase shifter. This involves the use of a linear array of voltage-controlled electronic oscillators coupled to nearest neighbors. The oscillators are mutually injection locked by controlling their coupling and tuning appropriately.[2][3] Stephan's approach consists of deriving two signals from a master oscillator, one signal phase shifted with respect to the other by means of a single phase shifter. These two signals are injected into the end oscillators of the array as shown in Figure 1. The result is a linear phase progression across the oscillator array. Thus, if radiating elements are connected to each oscillator and spaced uniformly along a line, they will radiate a beam at an angle to that line determined by the phase gradient which is, in turn, determined by the phase difference between the injection signals. The beam direction is therefore controlled by adjusting this phase difference.

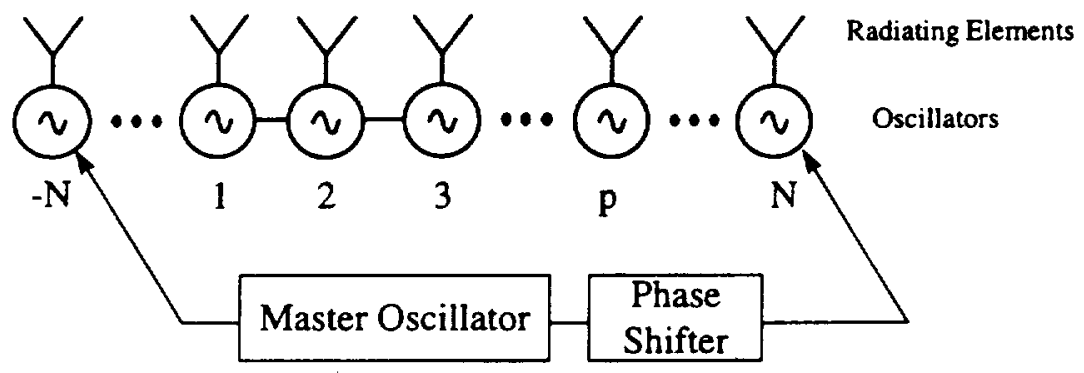

Figure 1. A one dimensional array of coupled oscillators with end injection.

Recently, Pogorzelski and York presented a formulation which facilitates theoretical analysis of the above beam steering technique [4][5]. This was subsequently applied by Pogorzelski in analysis of twodimensional beam steering using perimeter detuning of a coupled oscillator array [6]. The formulation is based on a continuum model in which the oscillator phases are represented by a continuous function satisfying a partial differential equation of diffusion type. This equation can be solved via the Laplace transform and the resulting solution exhibits the dynamic behavior of the array as the beam is steered.

Stephan's beam steering technique can be similarly generalized to two-dimensional arrays in which the beam control signals are applied to the oscillators on the perimeter of the array. In this paper the continuum model for this two-dimensional case is developed and the dynamic solution for the corresponding aperture phase function is obtained. The corresponding behavior of the resulting far-zone radiation pattern is displayed as well.

\section{The Continuum Model}

Figure 1 shows a two-dimensional array with signals injected into the perimeter oscillators for the purpose of steering the beam. Let a radiating element be connected to each oscillator of the array and let the elements be uniformly spaced on a planar surface. The aperture phase of this antenna is represented by a continuous function governed by the partial differential equation 


$$
\frac{\partial^{2} \phi}{\partial x^{2}}+\frac{\partial^{2} \phi}{\partial y^{2}}-V(x, y) \phi-\frac{\partial \phi}{\partial \tau}=-\frac{\omega_{\text {tune }}-\omega_{\text {ref }}}{\Delta \omega_{l o c k}}-V(x, y) \phi_{\text {inj }}(x, y ; \tau) \text {. }
$$

where $\phi$ is the aperture phase, $\phi_{\text {inj }}$ gives the phase of the injection signals, and $V$ describes the strength and distribution of the injection signals. $\tau$ is a dimensionless time measured in inverse inter-oscillator locking ranges. The dynamic behavior of the phase is obtained by solving this equation subject to Neumann boundary conditions on the periphery.

For perimeter injection, the function $V$ takes the form

where

$$
V(x, y)=C P(x)+C Q(y),
$$

and

$$
C=\frac{\Delta \omega_{i n j}}{\Delta \omega_{\text {lock }}}
$$

$$
\begin{aligned}
& C P(x)=\Omega_{x_{1}} \delta\left(x-x_{1}^{\prime}\right)+\Omega_{x_{2}} \delta\left(x-x_{2}^{\prime}\right), \\
& C Q(y)=\Omega_{y_{1}} \delta\left(y-y_{1}^{\prime}\right)+\Omega_{y_{2}} \delta\left(y-y_{2}^{\prime}\right) .
\end{aligned}
$$

The equation is solved by Laplace transformation and expansion of the Green's function in terms of the eigenfunctions of the differential operator, obtained by separation of variables, followed by integration over the sources represented by $V \phi_{\text {inj. }}$.

For beamsteering

$$
f_{i n j}(x, y ; s)=\frac{1}{s}\left[-x \sin \theta_{0} \cos \phi_{0}-y \sin \theta_{0} \sin \phi_{0}\right] \frac{2 \pi d}{\lambda} .
$$

Thus, $P(x) f_{\text {inj }}+Q(y) f_{\text {inj }}$ will be

$$
\begin{aligned}
P(x) f_{i n j}+Q(y) f_{i n j}= & \frac{1}{s}\left[-\delta\left(x-x_{1}^{\prime}\right) x_{1}^{\prime} \sin \theta_{0} \cos \phi_{0}-\delta\left(x-x_{1}^{\prime}\right) y \sin \theta_{0} \sin \phi_{0}\right. \\
& -\delta\left(x-x_{2}^{\prime}\right) x_{2}^{\prime} \sin \theta_{0} \cos \phi_{0}-\delta\left(x-x_{2}^{\prime}\right) y \sin \theta_{0} \sin \phi_{0} \\
& -\delta\left(y-y_{1}^{\prime}\right) x \sin \theta_{0} \cos \phi_{0}-\delta\left(y-y_{1}^{\prime}\right) y_{1}^{\prime} \sin \theta_{0} \sin \phi_{0} \\
& \left.-\delta\left(y-y_{2}^{\prime}\right) x \sin \theta_{0} \cos \phi_{0}-\delta\left(y-y_{2}^{\prime}\right) y_{2}^{\prime} \sin \theta_{0} \sin \phi_{0}\right] \frac{2 \pi d}{\lambda} .
\end{aligned}
$$

The required integrations can be carried out analytically owing to the delta functions in the integrand. Each term of the series for the solution has a pole in the $s$ plane, the location of which is determined by eigenvalue correponding to that term. Thus, the inverse Laplace transform can be obtained via residue calculus.

\section{A Numerical Example}

Consider a 21 by 21 element square array with radiating elements spaced one half wavelength apart. Injection signals are assumed to be applied to the perimeter oscillators with phases appropriate to steering the beam to far field coordinates $\theta_{0}=30^{\circ}$ and $\phi_{0}=-110^{\circ}$ switched at time zero. Figure 2 illustrates the ensuing dynamic behavior of the oscillators phases. 


\section{Oscillator Phases}

Two Omensional Array

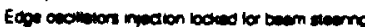
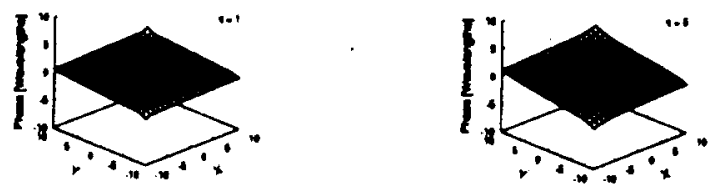

- 30 angeras

$1 . .110$ angre.
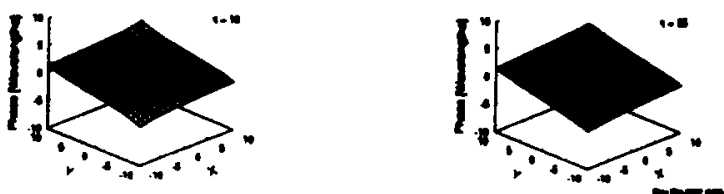

Figure 2. Phase dynamics during beam steering.

Note that during this transient period the phase surface is nonplanar which results in some aberration induced gain reduction and sidelobe distortion in the far-zone beam. Figure 3 shows the effect of this aberration on the far-zone gain as a function of time. The curve labeled "Ideal Gain" includes the projected aperture loss but no aberration loss for comparison.

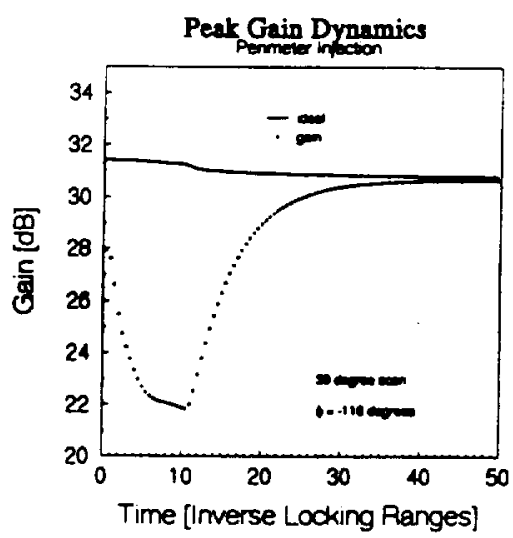

Figure 3. Antenna gain during beam steering.

Finally. Figure 4 shows the result of successive application of a sequence of beamsteering by displaying the locations of the beam peak and the three $\mathrm{dB}$ contour at a sequence of times and the effect of aberration on the beam shape is evident. 


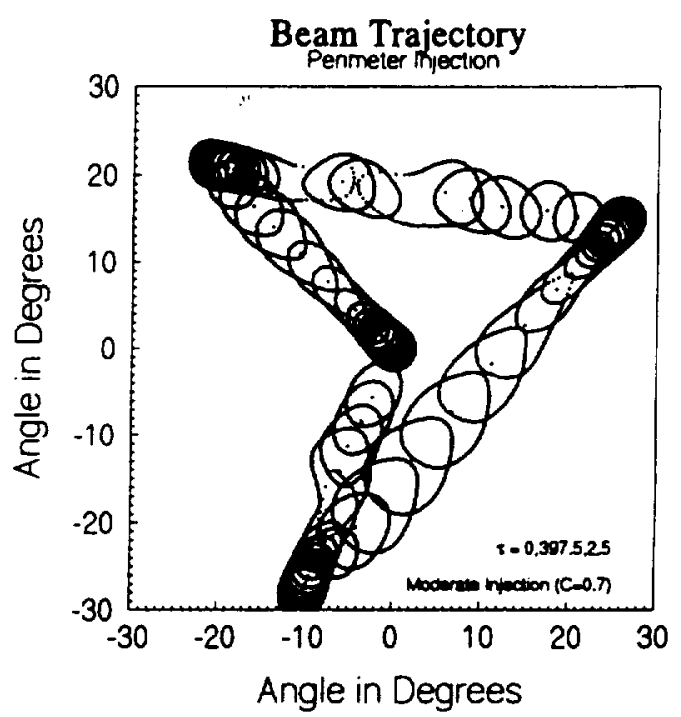

Figure 4. Sequential beam steering.

\section{Acknowledgment}

The research described in this paper was performed by the Center for Space Microelectronics Technology, Jet Propulsion Laboratory, California Institute of Technology, and was sponsored by the Ballistic Missile Defense Organization through an agreement with the National Aeronautics and Space Administration.

\section{$\underline{\text { References }}$}

1. K. D. Stephan, "Inter-Injection-Locked Oscillators for Power Combining and Phased Arrays," IEEE Trans. Microwave Theory and Tech., MTT-34, pp. 1017-1025, October 1986.

2. R. A. York, "Nonlinear Analysis of Phase Relationships in Quasi-Optical Oscillator Arrays," IEEE Trans. Microwave Theory and Tech., MTT-41, pp. 1799-1809, October 1993.

3. H.-C. Chang, E. S. Shapiro, and R. A. York, "Influence of the Oscillator Equivalent Circuit on the Stable Modes of Parallel-Coupled Oscillators," IEEE Trans. Microwave Theory and Tech., MTJ-45, pp. 1232-1239, August 1997.

4. R. J. Pogorzelski, P. F. Maccarini, and R. A. York, "A Continuum Model of the Dynamics of Coupled Oscillator Arrays for Phase Shifterless Beam-Scanning," submitted for publication in IEEE Trans. Microwave Theory and Tech.

5. R. J. Pogorzelski, P. F. Maccarini, and R. A. York, "Continuum Modeling of the Dynamics of Externally Injection Locked Coupled Oscillator Arrays," submitted for publication in IEEE Trans. Microwave Theory and Tech.

6. R. J. Pogorzelski, "On the Dynamics of Two-dimensional Amay Beam Scanning via Coupled Oscillators," submitted for publication in IEEE Trans. Antennas and Propagation. 


\title{
Two Dimensional Array Beam Scanning via Externally and Mutually Injection Locked Coupled Oscillators
}

\author{
R. J. Pogorzelski \\ Jet Propulsion Laboratory \\ California Institute of Technology \\ Pasadena, California
}

The research described in this paper wes performed by the Center for Space Microelectronics

Technology. Je Propulsion Laboriony. California Institute of Technolony, and was supported by the

Aallistic Miscile Defesse Oreanization through an agreement with the Nationd Aeronatics and Spece
Administontion. 


\section{Agenda}

- Introduction and Background

- The Continuum Model in Two Dimensions

- The Green's Function

- Solution for Beamsteering

- Example Beam Scanning Behavior

- Concluding Remarks

This presentation will begin with a description of the previous published work contributing to the results reported here. The previously developed one dimensional continuum model will be generalized to two dimensions and a Green's function for the resulting differential equation will be obtained as an eigenfunction expansion. This will be used to obtain dynamic solutions relevant to the steering of the radiated beam. Finally, some remarks concerning limitations on the interoscillator phase difference will be provided. 


\section{Introduction}

- Concept due to K. Stephan [IEEE Trans. MTT-34, pp. 1017-1025,October 1986].

- Linear array of mutually injection locked VCOs.

- External injection locking of end oscillators.

- Shift relative phase of injection signals.

- Linear aperture phase with variable gradient.

- Analysis via numerical solution of a system of first order nonlinear differential equations based on Adler's theory of injection locking.

The fundamental concept of steering phased array beams by appropriately injection locking the end oscillators of a linear array originated with Karl Stephan circa 1986. He suggested that linear phase progressions alon the array could be established if the end oscillators were injection locked to a common external source and a phase shifter were inserted in one line to control the relative phase of the two injection signals.

This analysis of the array took the form of numerical solution of a system of first order nonlinear differential equations derived using Adler's theory of injection locking. This made intuitive understanding of the dynamics difficult. 


\section{Stephan's Beamsteering Scheme}

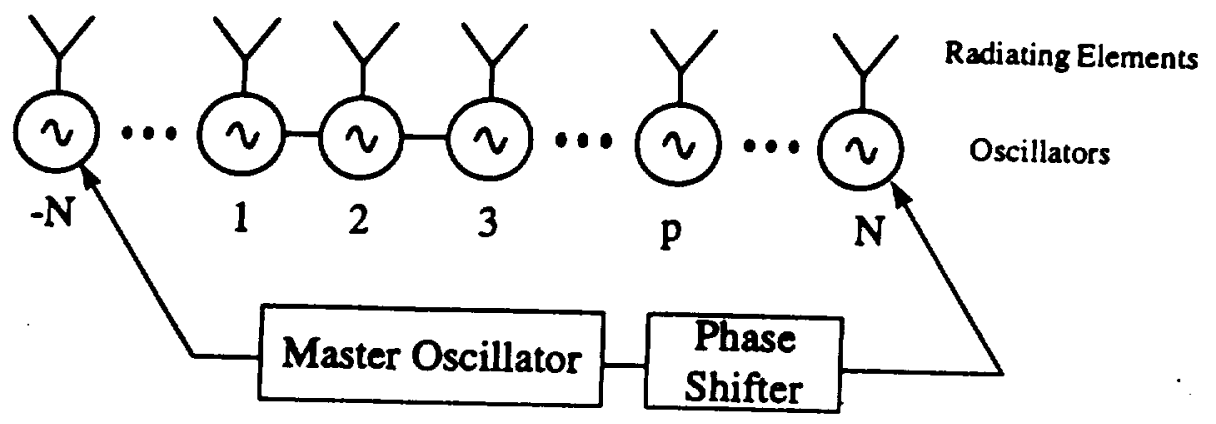

This diagram shows the Stephan scheme for beam steering. The master oscillator provides injection signals to the two end oscillators while the phase shifter controls the relative phase of these signals. The result is a linear phase progression across the array. 


\section{Introduction (Continued)}

- Continuum model by Pogorzelski and York [IEEE AP-S Symposium Digest, pp. 324327, July 1997].

- Continuous phase function of continuous variable indexing oscillators.

- Governed by second order partial differential equation.

- Steady state is analogous to electrostatics.

- Detuning=Charge

- Phase $=$ Potential

Beginning around 1996, Pogorzelski and York developed a continuum model of coupled oscillator arrays in which the phase is described by a continuous function of a continuous variable which, when it takes on integer values, indexes the oscillators of the array. The behavior of this continuous function is governed by a second order linear partial differential equation which can be solved analytically using standard techniques. This greatly enhances insight into the dynamics of such arrays and the relationship between the behavior of the phase and the external injection signals. 


\section{The $\mathrm{M}$ by $\mathrm{N}$ Array}

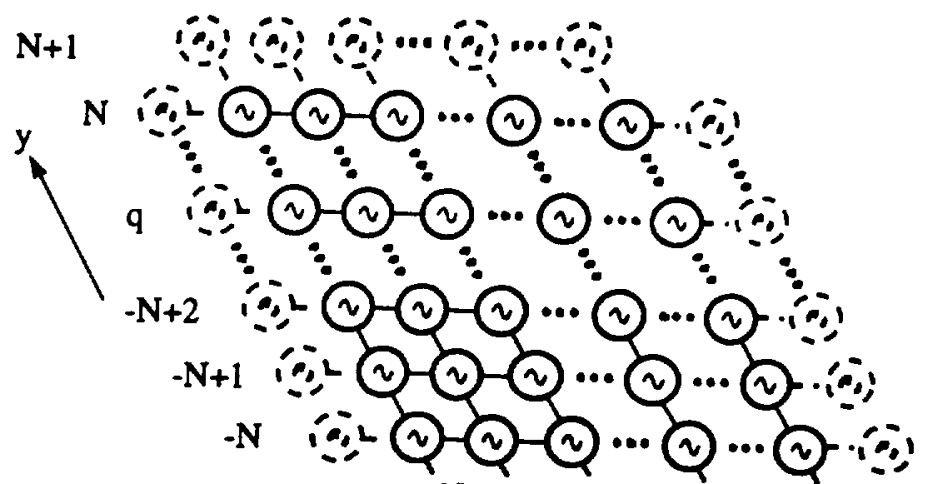

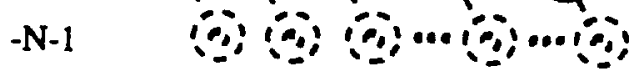

$-M-1-M-M+1-M+2 \quad p \quad M \quad M+1$

This diagram schematically represents a $(2 \mathrm{M}+1)$ by $(2 \mathrm{~N}+1)$ array of oscillators coupled to nearest neighbors. This is the array to be analyzed in the following. The oscillators shown in dashed lines are external sources which provide the properly phased injection signals to the perimeter oscillators of the array. 


\section{The Continuum Model}

- Begin with Adler's theory applied to the array.

$$
\begin{aligned}
\frac{d G_{i j}}{d t}=\omega_{\text {nuncij } i j} & -\sum_{m=-M}^{M} \sum_{n=-N}^{N} \Delta \omega_{l o c k: i j, m n} \sin \left(\Phi_{i j, m n}+\theta_{i j}-\theta_{m n}\right) \\
& -\sum_{p=-M}^{M} \sum_{q=-N}^{N} \Delta \omega_{i j ;: j . p q} \sin \left(\Phi_{i j . p q}+\theta_{i j}-\theta_{p q}\right)
\end{aligned}
$$

Define the phase by:

$$
\theta_{i j}=\omega_{r e f} t+\phi_{i j}
$$

To derive the continuum model of this two dimensional array, we begin with Adler's description of the injection locking phenomenon. In his theory, the time derivative of the phase of an injection locked oscillator is related to the sine of the phase difference between the oscillator signal and the injection signal. Generalizing this to the two dimensional array of mutually injection locked oscillators (with general interoscillator coupling topology) we arrive at the system of differential equations shown. We then define the phase, phi, as shown relative to a reference frequency which can be chosen arbitrarily.

The second double summation represents the externally provided injection signals. 


\section{The Continuum Model (Cont.)}

Then,

$$
\begin{aligned}
& \frac{d \phi_{i 1}}{d t}=\omega_{\text {neme ij }}-\omega_{r e d}-\sum_{\substack{m=i-1 \\
m=i}}^{i+1} \sum_{\substack{n=j-1 \\
n \in j}}^{i+1} \Delta \omega_{\text {lock } i j / m n} \sin \left(\Phi_{i j, m n}+\phi_{i j}-\phi_{m n}\right) \\
& -\sum_{p=-M}^{M} \sum_{q=-N}^{N} \Delta \omega_{i n j j j, p q} \sin \left(\Phi_{i j, p q}+\phi_{i j}-\phi_{p q}\right)
\end{aligned}
$$

or,

$$
\begin{gathered}
\frac{d \phi_{i j}}{d t}=\omega_{\text {aine }, j}-\omega_{\text {ref }}+\Delta \omega_{\text {lock }}\left[\phi_{i-1, j-1}\right. \\
\left.+\phi_{i-1, j+1}+\phi_{i+1, j-1}+\phi_{i+1, j+1}-4 \phi_{i j}\right] \\
-\sum_{q=-M}^{M} \sum_{p=-N}^{N} \Delta \omega_{i n j}\left(\phi_{i j}-\phi_{p q}\right)
\end{gathered}
$$

Using this definition of phi the system of equations become that shown here. Then, assuming that the locking ranges are all the same, that the coupling phase is zero, and that the phase differences between adjacent oscillators is small, we can linearize the system as shown. Then, the quantity in the square brackets can be identified as the finite difference approximation to the Laplacian operator. 


\section{The Continuum Model (Cont.)}

which leads to,

$\frac{\partial^{2} \phi}{\partial x^{2}}+\frac{\partial^{2} \phi}{\partial y^{2}}-V(x, y) \phi-\frac{\partial \phi}{\partial \tau}=-\frac{\omega_{\text {rune }}-\omega_{\text {ref }}}{\Delta \omega_{\text {lock }}}-V(x, y) \phi_{\text {inj }}(x, y ; \tau)$

where,

$$
\tau=\Delta \omega_{\text {lock }} t
$$

Thus, defining a continuous phi function and continuous variables $x$ and $y$ indexing the oscillators, we arrive at the partial differential equation for phi shown. $V$ represents the distribution and strength of the injection signals with phase $\mathrm{phi}_{\mathrm{inj}}$. Tau is time measured in inverse locking ranges. 


\section{Boundary Conditions}

- Employ an artifice proposed in the one dimensional case.

- Add fictitious oscillators on the periphery.

- Dynamically tuned to reduce injection to zero.

- Results in a Neumann condition on the boundary.

Having derived the differential equation governing the behavior of phi, we must determine the boundary conditions at the perimeter of the array in order to uniquely define the solution. For this we use an artifice in which fictitious oscillators are added on the periphery of the array and these are dynamically tuned in such a manner as to render the phase of each fictitious oscillator equal to its nearest real neighbor in the array. This effectively emulates the absence of the fictitious oscillator because when the phases are equal the injection effect on the dynamics is zero by Adler's theory. Now, the equality of the two phases implies a zero value for the derivative of phase normal to the array edge; i.e., a Neumann boundary condition. 


\section{The $\mathrm{M}$ by $\mathrm{N}$ Array}

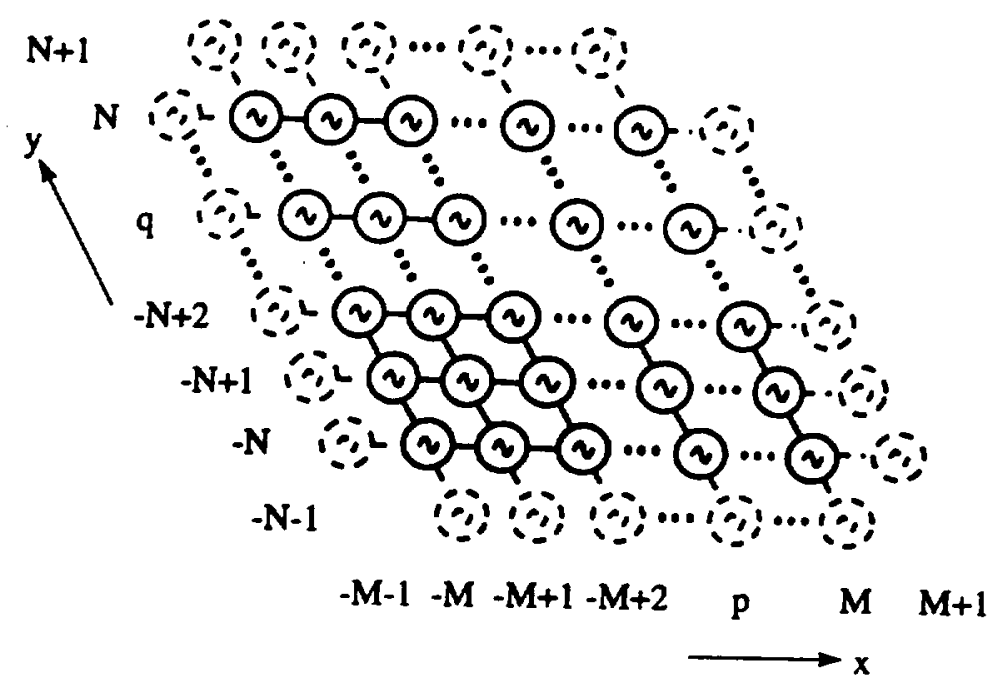

This diagram illustrates the fictitious oscillator arrangement used in the boundary condition derivation. 


\section{Injection of Perimeter Oscillators}

$$
\begin{gathered}
V(x, y)=C P(x)+C Q(y) \\
C=\frac{\Delta \omega_{i n j}}{\Delta \omega_{\text {lock }}} \\
C P(x)=\Omega_{x_{1}} \delta\left(x-x_{1}^{\prime}\right)+\Omega_{x_{2}} \delta\left(x-x_{2}^{\prime}\right) \\
C Q(y)=\Omega_{y_{1}} \delta\left(y-y_{1}^{\prime}\right)+\Omega_{y_{2}} \delta\left(y-y_{2}^{\prime}\right)
\end{gathered}
$$

We intend to supply externally derived injection signals only to the oscillators on the perimeter of the array. This implies that the function $F$ takes the form shown. $C$ measures the strength of the injection signals and is measured in terms of the implied locking rangerelative to the inter-oscillator locking range. 


\section{The Differential Equation}

$$
\begin{aligned}
\frac{\partial^{2} \phi}{\partial x^{2}} & +\frac{\partial^{2} \phi}{\partial y^{2}}-C P(x) \phi-C Q(y) \phi-\frac{\partial \phi}{\partial \tau} \\
& =-C P(x) \phi_{i n j}(x, y ; \tau)-C Q(y) \phi_{i n j}(x, y ; \tau)
\end{aligned}
$$

For the case of perimeter injection, the differential equation governing the phase dynamics takes the form shown. This equation will be solved by means of a Green's function, G; that is, a solution for the case of a delta function source term. The Green's function will be expressed as a sum of the eigenfunctions of the operator in the classical manner. 


\section{The Homogeneous Equation}

$$
\frac{\partial^{2} \phi}{\partial x^{2}}+\frac{\partial^{2} \phi}{\partial y^{2}}-C P(x) \phi-C Q(y) \phi-\frac{\partial \phi}{\partial \tau}=0
$$

$$
\frac{\partial^{2} f}{\partial x^{2}}+\frac{\partial^{2} f}{\partial y^{2}}-C P(x) f-C Q(y) f-s f=0
$$

We begin by consideration of the homogeneous equation. The Laplace transform with respect to time is shown at the bottom of the slide. 


\section{Separation of Variables}

Let $f(x, y)=X\left(x, s_{x}\right) Y\left(y, s_{y}\right)$.

Then,

$$
\begin{aligned}
& X^{\prime \prime}-C P X-s_{x} X=0 \\
& Y^{\prime \prime}-C Q Y-s_{y} Y=0
\end{aligned}
$$

where, $s=s_{x}+s_{y}$.

$$
\begin{aligned}
& X^{\prime \prime}-\Omega_{x_{1}} \delta\left(x-x_{1}^{\prime}\right)-\Omega_{x_{2}} \delta\left(x-x_{2}^{\prime}\right)-s_{x} X=0 \\
& Y^{\prime \prime}-\Omega_{y_{1}} \delta\left(y-y_{1}^{\prime}\right)-\Omega_{y_{2}} \delta\left(y-y_{2}^{\prime}\right)-s_{y} Y=0
\end{aligned}
$$

The homogeneous equation can be solved by the classical separation of variables method. We assume a product solution and obtain two ordinary second order differential equations for the $x$ and $y$ dependences of the solution. Substituting the deisred form of the $\mathrm{V}$ function results in the equations shown at the bottom
of the slide. 


\section{The Solution for X}

$$
\begin{aligned}
X= & A_{1} \cosh \left[\sqrt{s_{1}}\left(a+\frac{1}{2}+x\right)\right] \cosh \left[\sqrt{s_{s}}\left(a+\frac{1}{2}-x_{1}^{\prime}\right)\right] \\
& +A_{2} \cosh \left[\sqrt{s_{x}}\left(a+\frac{1}{2}+x\right)\right] \cosh \left[\sqrt{s_{x}}\left(a+\frac{1}{2}-x_{2}^{\prime}\right)\right] ;-a-\frac{1}{2} \leq x \leq x_{1}^{\prime} \\
X= & A_{1} \cosh \left[\sqrt{s_{x}}\left(a+\frac{1}{2}-x\right)\right] \cosh \left[\sqrt{s_{x}}\left(a+\frac{1}{2}+x_{1}^{\prime}\right)\right] \\
& +A_{2} \cosh \left[\sqrt{s_{x}}\left(a+\frac{1}{2}+x\right)\right] \cosh \left[\sqrt{s_{1}}\left(a+\frac{1}{2}-x_{2}^{\prime}\right)\right] ; \quad x_{2}^{\prime} \leq x \leq x_{1}^{\prime} \\
X= & A_{1} \cosh \left[\sqrt{s_{x}}\left(a+\frac{1}{2}-x\right)\right] \cosh \left[\sqrt{s_{x}}\left(a+\frac{1}{2}+x_{1}^{\prime}\right)\right] \\
& +A_{2} \cosh \left[\sqrt{s_{x}}\left(a+\frac{1}{2}-x\right)\right] \cosh \left[\sqrt{s_{x}}\left(a+\frac{1}{2}+x_{2}^{\prime}\right) ; \quad x_{2}^{\prime} \leq x \leq a+\frac{1}{2}\right.
\end{aligned}
$$

Focussing now on the $x$ dependence, we find that the solution can be expressed in the form shown with two unknown constants, the A's. The A's can be determined by inposing the appropriate discontinutity condition on the spatial derivative at the location of the two delta functions. 


\section{Evaluation of the A's}

At the delta's,

$$
\begin{aligned}
& \left.X^{\prime}\right|_{x_{1}^{\prime}} ^{x_{1}^{*}}=\Omega_{x_{1}} X\left(x_{1}^{\prime}\right) \\
& \left.X^{\prime}\right|_{x_{2}^{\prime-}} ^{x_{2}^{\prime *}}=\Omega_{x_{2}} X\left(x_{2}^{\prime}\right)
\end{aligned}
$$

which yields,

$$
\left[\begin{array}{ll}
M_{11} & M_{12} \\
M_{21} & M_{22}
\end{array}\right]\left[\begin{array}{l}
A_{1} \\
A_{2}
\end{array}\right]=0
$$

The discontinuity conditions are shown here. These, when applied to the solution, provide a homogeneous set of simultaneous linear equations for the A's. For a solution to exist, the determinant of the coefficients matrix must be zero. 


\section{Evaluation of the A's (Cont.)}

where,

$$
\begin{aligned}
& M_{11}=\sqrt{s_{x}} \sinh \left[\sqrt{s_{x}}(2 a+1)\right]+\Omega_{x_{1}} \cosh \left[\sqrt{s_{x}}\left(a+\frac{1}{2}+x_{i}^{\prime}\right)\right] \cosh \left[\sqrt{s_{x}}\left(a+\frac{1}{2}-x_{1}^{\prime}\right)\right] \\
& M_{12}=\Omega_{x_{1}} \cosh \left[\sqrt{s_{x}}\left(a+\frac{1}{2}+x_{1}^{\prime}\right)\right] \cosh \left[\sqrt{s_{x}}\left(a+\frac{1}{2}-x_{2}^{\prime}\right)\right] \\
& M_{21}=\Omega_{x_{2}} \cosh \left[\sqrt{s_{x}}\left(a+\frac{1}{2}+x_{1}^{\prime}\right)\right] \cosh \left[\sqrt{s_{x}}\left(a+\frac{1}{2}-x_{z}^{\prime}\right)\right] \\
& M_{22}=\sqrt{s_{x}} \sinh \left[\sqrt{s_{x}}(2 a+1)\right]+\Omega_{x_{2}} \cosh \left[\sqrt{s_{x}}\left(a+\frac{1}{2}+x_{2}^{\prime}\right)\right] \cosh \left[\sqrt{s_{x}}\left(a+\frac{1}{2}-x_{2}^{\prime}\right)\right]
\end{aligned}
$$

This slide provides the detailed analytic expressions for the matrix elements involved in the determinant. 


\section{The Eigenvalues, $s_{x}$}

$$
\begin{aligned}
& \Delta=\sinh \left[\sqrt{s_{x}}(2 a+1)\right] \int s_{x} \sinh \left[\sqrt{s_{x}}(2 a+1)\right]+\frac{1}{2}\left(\Omega_{x_{1}}+\Omega_{x_{1}}\right) \sqrt{s_{x}} \cosh \left[\sqrt{s_{x}}(2 a+1)\right] \\
& +\frac{1}{2} \Omega_{x_{1}} \sqrt{s_{x}} \cosh \left[\sqrt{s_{x}}\left(2 x_{1}^{\prime}\right)\right]+\frac{1}{2} \Omega_{x_{1}} \sqrt{s_{x}} \cosh \left[\sqrt{s_{x}}\left(2 x_{2}^{\prime}\right)\right] \\
& \left.-\frac{1}{2} \Omega_{x_{1}} \Omega_{x_{1}} \sinh \left[\sqrt{s_{x}}\left(x_{1}^{\prime}-x_{2}^{\prime}\right)\right]\left\{\cosh \left[\sqrt{s_{x}}\left(2 a+1+x_{1}^{\prime}-x_{2}^{\prime}\right)\right]+\cosh \left[\sqrt{s_{x}}\left(x_{1}^{\prime}+x_{2}^{\prime}\right)\right]\right\}\right)
\end{aligned}
$$

The determinant of the matrix of M's can be reduced to the form shown here. Setting this determinant equal to zero provides a transendental equation for the eigenvalues of $s_{x}$. 


\section{Eigenfunctions}

$$
\begin{array}{lll}
A_{1}=M_{12}=A_{1}^{(1)} & & A_{1}=M_{12}=A_{1}^{(1)} \\
A_{2}=-M_{22}=A_{2}^{(1)} & \text { or } & A_{2}=-M_{22}=A_{2}^{(1)}
\end{array}
$$

yields

$$
\begin{aligned}
& X\left(x, s_{s}\right)=\cosh \left[\sqrt{s_{x}}\left(2 h_{1}-\left|x_{2}^{\prime}-x\right|\right)\right]+\eta \cosh \left[\left(\sqrt{s_{1}}\left(2 h_{x}-\left|x_{1}^{\prime}-x\right|\right)\right]\right. \\
& \cosh \left[\sqrt{s_{s}}\left(x+x_{2}^{\prime}\right)\right]+\eta \cosh \left[\sqrt{s_{s}}\left(x+x_{1}^{\prime}\right)\right] \\
& \Omega_{4} \cosh \left[\sqrt{s_{x}}\left(h_{x}+x_{1}^{\prime}\right)\right\}\left\{\frac { 1 } { \sqrt { s _ { x } } } \operatorname { s i n h } \left\{\left[\sqrt{s_{x}}\left(h_{z}-x_{1}^{\prime}-\left|x_{2}^{\prime}-x\right|\right)\right]-\frac{1}{\sqrt{s_{x}}} \sinh \left\{\left[\sqrt{s_{x}}\left(h_{z}-x_{2}^{\prime}-\left|x_{1}^{\prime}-x\right|\right)\right]\right\}\right.\right. \text {. } \\
& \Omega_{h_{2}} \cosh \left[\sqrt{s_{x}}\left(h_{x}-x_{z}^{\prime}\right)\right\}\left\{\frac{1}{\sqrt{s_{s}}} \sinh \left[\sqrt{s_{x}}\left(h_{x}+x_{2}^{\prime}-\mid x_{1}^{\prime}-x\right)\right]-\frac{1}{\sqrt{s_{x}}} \sinh \left[\sqrt{s_{x}}\left(h_{x}+x_{1}^{\prime}-\left|x_{z}^{\prime}-x\right|\right)\right]\right\} \eta
\end{aligned}
$$

Once the eigenvalues are found, the homogeneous equations represent two relationships between the A's, each of which give a solution. These two solutions differ only by a multiplicative constant. However, this constant can, in certain cases, be zero for one of the solutions, indicating that the appropriate normalization constant is infinite. In such cases it is expedient to use the other solution.

The solution shown is, in fact, a linear combination of the two solution chosen to create a symmetric looking expression. For normalization reasons as described above, two options are represented; I.e., eta equal to one or minus one. The normalization integrals of the square of this function over the extent of the array can be carried out analytically because they only involve products of hyperbolic functions of $x$. The actual expressions are, however, a bit cumbersome. 


\section{Eigenfunctions (Cont.)}

- Similar procedure yields the y dependent eigenfunctions.

- The Green's function can now be written in terms of these two sets of functions.

- The Green's function has no direct physical significance in this instance.

- It will be used to obtain the physically meaningful solution.

Carrying out the same solution procedure for $y$ yields the set of $y$ dependent eigenfunctions. Multiplying the $\mathrm{x}$ and $\mathrm{y}$ dependent functions gives a doubly infinite set of normalized two dimensional eigenfunctions in terms of which the Green's function may be expressed.

It is noted that this Green's function is the solution of the differential equation with a delta function source. However, because the equation we are solving has line sources instead of point sources, it is necessary to integrate the Green's function over the sources to obtain a physically meaningful expression for the phase. 


\section{The Green's Function}

$$
G\left(x, y ; x^{\prime}, y^{\prime} ; s\right)=\sum_{n=1}^{\infty} \sum_{m=1}^{-} \frac{X\left(x^{\prime}, s_{m}\right) Y\left(y^{\prime}, s_{n}\right) X\left(x, s_{m}\right) Y\left(y, s_{n}\right)}{s-s_{m}-s_{n}}
$$

is a solution of,

$$
\frac{\partial^{2} \phi}{\partial x^{2}}+\frac{\partial^{2} \phi}{\partial y^{2}}-C P(x) \phi-C Q(y) \phi-\frac{\partial \phi}{\partial \tau}=\delta\left(x-x^{\prime}\right) \delta\left(y-y^{\prime}\right)
$$

This is the classical series expression for the Green's function in terms of the normalized eigenfunctions and eigenvalues. It is a solution of the unphysical equation shown at the bottom of the slide. It is unphysical because the source distribution on the right side does not match that described by $\mathrm{P}$ and $\mathrm{Q}$ on the
left side. 


\section{The Dynamic Solution}

We wish to solve,

$\frac{\partial^{2} f}{\partial x^{2}}+\frac{\partial^{2} f}{\partial y^{2}}-C P(x) f-C Q(y) f-s f=-C P(x) f_{i n j}(x, y ; s)-C Q(y) f_{i n j}(x, y ; s)$

In terms of the Green's function, the solution is,

$$
f(x, y ; s)=-C \int_{\left(-\infty-\frac{1}{2}\right)}^{\left(\infty-\frac{1}{2}\right)} \int_{\left(-\infty-\frac{1}{2}\right)}^{\left(-+\frac{1}{2}\right)} G\left(x, y ; x^{\prime}, y^{\prime} ; s\right)\left[P\left(x^{\prime}\right) f_{i v}\left(x^{\prime}, y^{\prime} ; s\right)+Q\left(x^{\prime}\right) f_{i v j}\left(x^{\prime}, y^{\prime} ; s\right)\right] d x^{\prime} d y^{\prime}
$$

To obtain the solution for the dynamic behavior of the oscillator phases, we must solve the partial differential equation shown. The Green's function is useful in that the integral shown at the bottom of the slide provides the solution sought. The integrand involves delta function and, as such, can be integrated quite easily. 


\section{$\mathrm{P}, \mathrm{Q}$, and $\mathrm{f}_{\text {inj }}$ for Beamsteering}

$$
\begin{aligned}
f_{i n j}(x, y ; s)= & \frac{1}{s}\left[-x \sin \theta_{0} \cos \phi_{0}-y \sin \theta_{0} \sin \phi_{0}\right] \frac{2 \pi d}{\lambda} \\
P(x) f_{i v}+Q(y) f_{w j}= & \frac{1}{s}\left[-\delta\left(x-x_{1}^{\prime}\right) x_{1}^{\prime} \sin \theta_{0} \cos \phi_{0}-\delta\left(x-x_{1}^{\prime}\right) y \sin \theta_{0} \sin \phi_{0}\right. \\
& -\delta\left(x-x_{2}^{\prime}\right) x_{2}^{\prime} \sin \theta_{0} \cos \phi_{0}-\delta\left(x-x_{2}^{\prime}\right) y \sin \theta_{0} \sin \phi_{0} \\
& -\delta\left(y-y_{1}^{\prime}\right) x \sin \theta_{0} \cos \phi_{0}-\delta\left(y-y_{1}^{\prime}\right) y_{1}^{\prime} \sin \theta_{0} \sin \phi_{0} \\
& \left.-\delta\left(y-y_{2}^{\prime}\right) x \sin \theta_{0} \cos \phi_{0}-\delta\left(y-y_{2}^{\prime}\right) y_{2}^{\prime} \sin \theta_{0} \sin \phi_{0}\right] \frac{2 \pi d}{\lambda}
\end{aligned}
$$

A important consideration at this point is the determination of the necessary injection signal phasing for beamsteering. We postulate the form indicated at the top of the slide. Using the $P$ and $Q$ appropriate to perimeter injection we obtain the expression shown at the bottom of the slide. This is linear in $x$ and $y$ and, as a result, it can be shown that $f_{\text {inj }}$ represents the steady state solution for the phase. It is also just that necessary to steer the beam to the angles indicated. 


\section{The Dynamic Beamsteering Phase Solution}

$$
\phi(x, y ; \tau)=\sum_{n=1}^{\infty} \sum_{m=1}^{\infty} R_{m n}(x, y)\left[1-e^{\left(s_{m}+s_{n}\right) \tau}\right] \mu(\tau)
$$

which converges slowly. The convergence rate can be improved by adding and subtracting the known steady state solution. That is,

Substituting the beamsteering source function into the integral expression for the solution and carrying out the integrations, which can be done analytically, we obtain the series expansion for the solution. Each term of the series has a pole in the complex plane the location of which is given by the eigenvalue corresponding to that term. The inverse Laplace transform can thus be obtained by residue calculus and the solution in the time domain expressed as the residue series shown. 


\section{The Dynamic Beamsteering Phase Solution (Cont.)}

$$
\begin{aligned}
\phi(x, y ; \infty) & =\sum_{n=1} \sum_{m=1} R_{m i}(x, y) u(\tau)=\phi_{i v j}(x, y) \\
& =\lim _{s \rightarrow 0}\left\{s f_{i n j}(x, y, s)\right\}=-\left(x \sin \theta_{0} \cos \phi_{0}+y \sin \theta_{0} \sin \phi_{0}\right) \frac{2 \pi d}{\lambda}
\end{aligned}
$$

and the solution may be written in the form,

$$
\phi(x, y ; \tau)=\sum_{n=1} \sum_{m=1} R_{m}(x, y)\left[-e^{\left(x_{n}+s_{0}\right) r}\right) \mu(\tau)-\left(x \sin \theta_{0} \cos \phi_{0}+y \sin \theta_{0} \sin \phi_{0}\right) \frac{2 \pi d}{\lambda}
$$

The rate of convergence of the residue series can be increased by subtracting the steady state value of the series terms and then adding the known steady state solution to the result. This procedure is illustrated in this slide. 


\section{A Numerical Example}

- Consider a 21 by 21 element square array.

- Radiating elements:

- Half wavelength spacing

- Connected to each oscillator

Consider a 21 by 21 element array with one radiating element connected to each oscillator. Let the radiating elements be spaced one half wavelength apart and let the external injection signals be applied to the perimeter oscillators per the preceding theory. The following vugraphs show a series of computed results concerning the aperture phase and far zone field of such an array. 


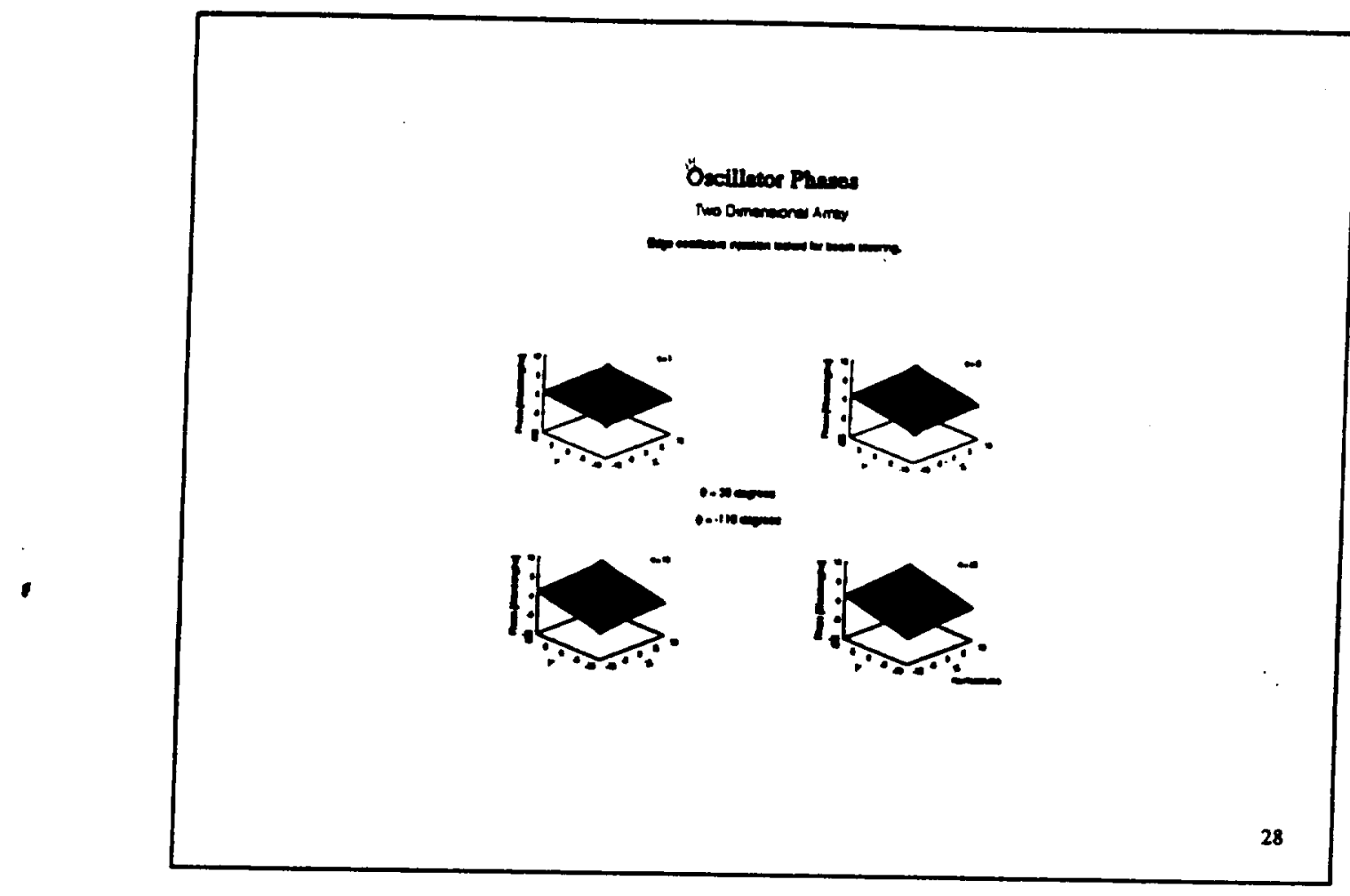

These graphs show the time evolution of the phase when injection phase appropriate to beamsteering is applied. 


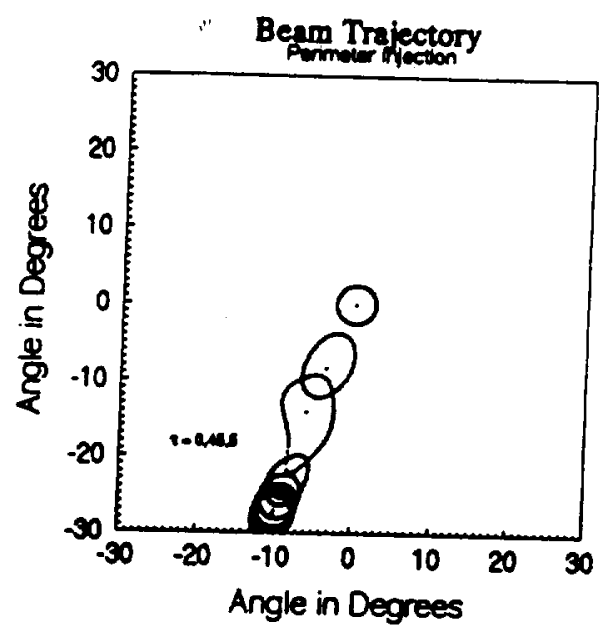

29

This graph shows the beam peak (dots) and the three $\mathrm{dB}$ contour (closed curves) as a function of time during the beamsteering transient resulting when a step steering injection phase designed to steer the beam thirty degrees off normal is applied at time zero. 


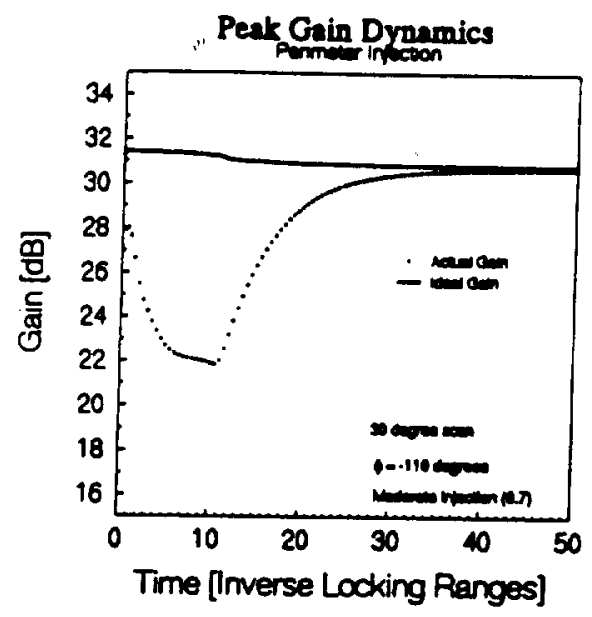

During the transient period, the aperture phase is nonplanar. This results in a temporary reduction in gain due to phase aberration. This graph shows this gain reduction as a function of time compared with the projected aperture loss to be expected for each beam position. These curves were obtained by pattern integration.

The irregular behavior is attributed to the fact that the abscissa is time as opposed to angle. Thus, the irregularities are due to changes in the rate of beam motion. 


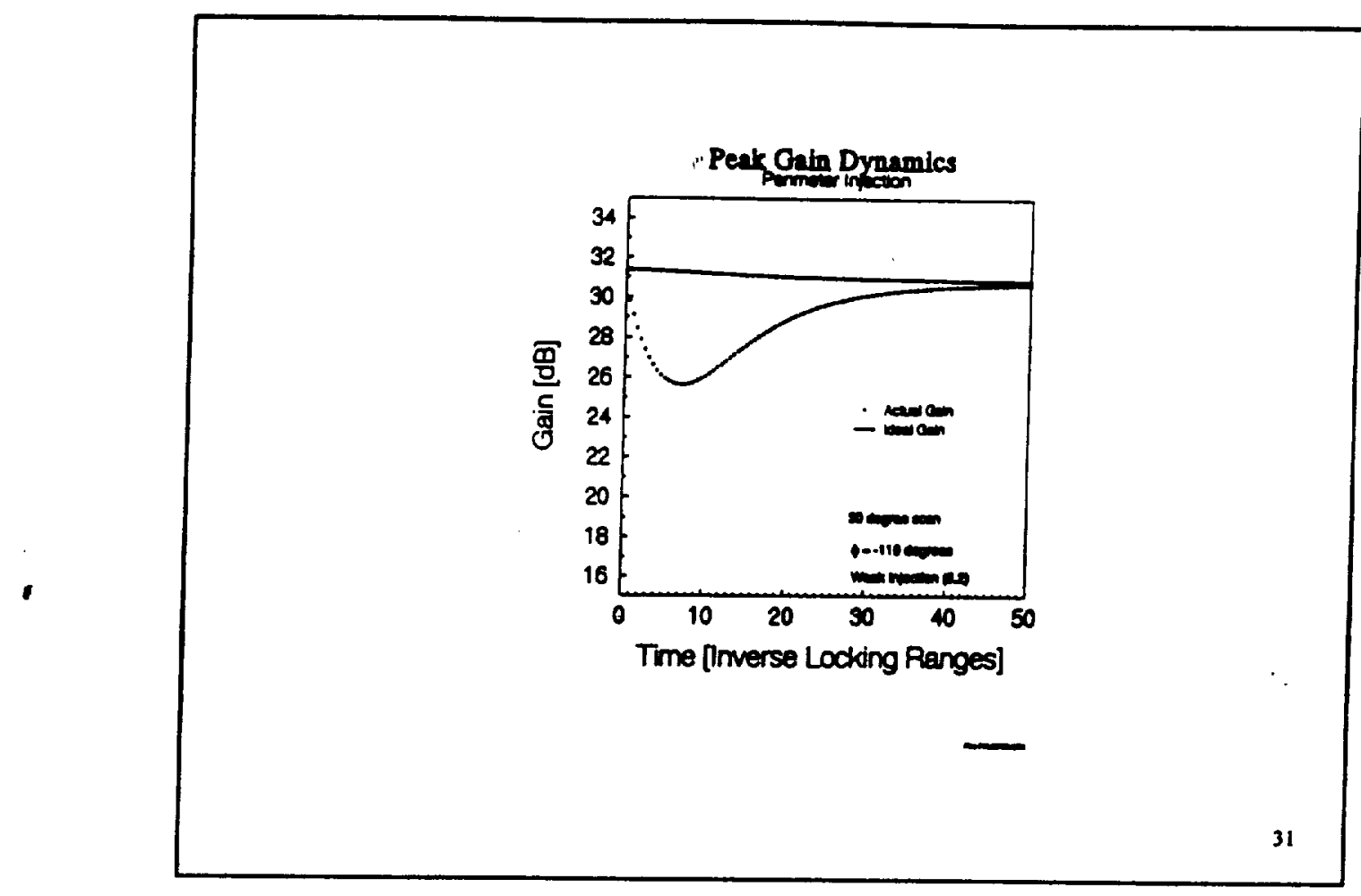

This shows a result corresponding to the one shown in the previous slide but, this time, with a weaker injection signal level. Note that this causes the transient to decay more slowly. 


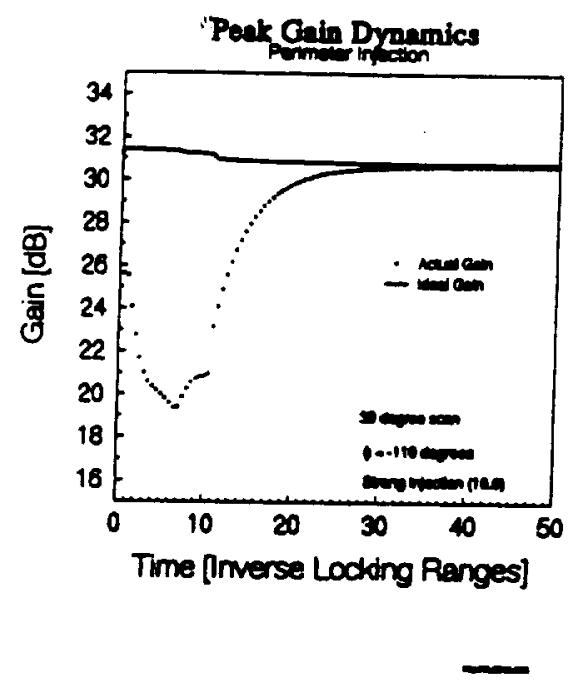

This shows a result corresponding to the one shown in the previous slide but, this time, with a stronger injection signal level. Note that, while this causes the transient to decay more rapidly, the gain dip is deeper and the peak moves more erratically in this case. 


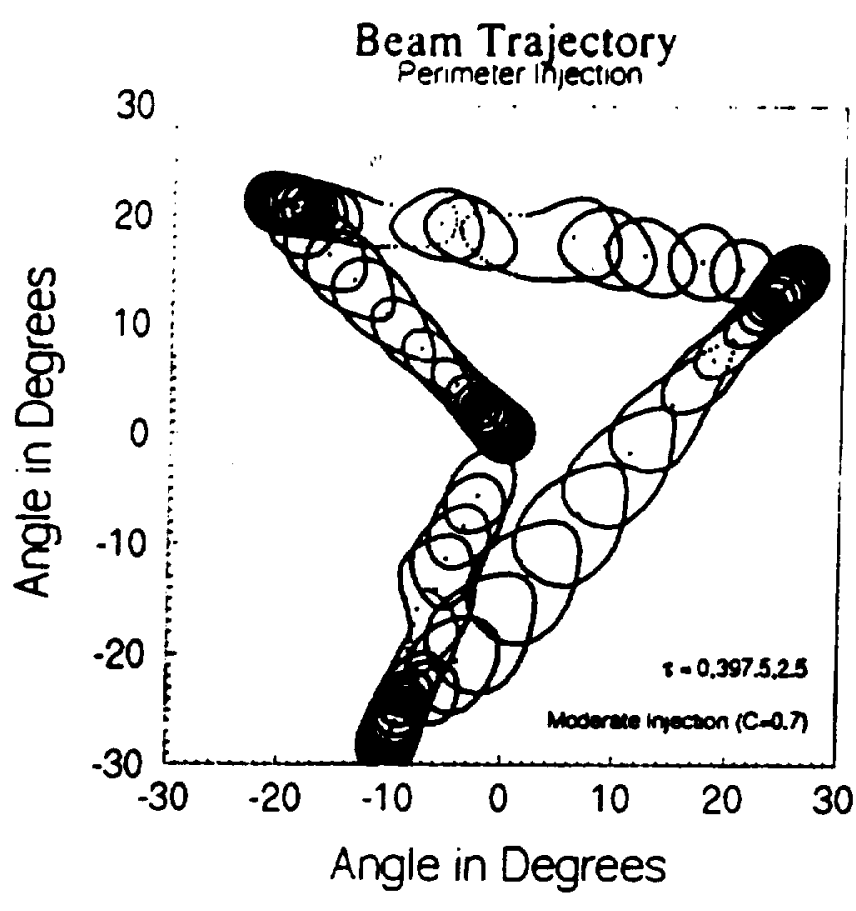

This graph shows the result of four sets of steering injection phases applied in rapid succession. Note that the aberration effects seem to be greater when steering from one off axis position to another than when steering to or from normal. 


\section{Concluding Remarks}

- Inter-oscillator phase difference

- Limited to 90 degrees.

- Limit can be mitigated by:

- Reducing the element spacing.

- Adding oscillators between the radiating ones.

- Radiating at a harmonic of the coupling frequency.

- Injection signal phase limited to 90 degrees from injected oscillator phase unless applied gradually.

One limitation of the present system is that the phase difference between adjacent oscillators is limited to 90 degrees to maintain lock. (The validity of the linearized theory actually requires that the phase difference be small compared to 90 degrees.) This would appear to limit the scan of a radiating aperture with half wavelength element spacing to 30 degrees off axis. However, this can be mitigated in several ways. One can reduce the spacing between the elements, one can radiate only from every second or every third oscillator, or one can radiate at a harmonic of the coupling frequency.

Another apparent limitation concerns the injection signal phase. This arises because, to maintain lock, the injection phase cannot differ from the injected oscillator phase by more than 90 degrees. However, in practice this limitation is easily circumvented because it only applies to suddenly applied steering phases. If the phase is applied gradually, the only limit is the one discussed above. 